\title{
Short-term impact of sucralose consumption on the metabolic response and gut microbiome of healthy adults
}

\author{
Pamela Thomson $^{1} \uparrow$, Rodrigo Santibañez ${ }^{1} \dagger$, Carolina Aguirre ${ }^{2}$, Jose E. Galgani ${ }^{2,3}$ and Daniel Garrido ${ }^{1 *}$ \\ ${ }^{1}$ Department of Chemical and Bioprocess Engineering, School of Engineering, Pontificia Universidad Católica de Chile, \\ Santiago, Chile \\ ${ }^{2}$ Departamento Ciencias de la Salud, Carrera de Nutrición y Dietética, Facultad de Medicina, Pontificia Universidad Católica \\ de Chile, Santiago, Chile \\ ${ }^{3}$ Departamento de Nutrición, Diabetes y Metabolismo, Facultad de Medicina, Pontificia Universidad Católica de Chile, \\ Santiago, Chile \\ (Submitted 19 March 2019 - Final revision received 8 June 2019 - Accepted 21 June 2019 - First published online 13 September 2019)
}

\section{Abstract}

Sucralose is an artificial non-nutritive sweetener used in foods aimed to reduce sugar and energy intake. While thought to be inert, the impact of sucralose on metabolic control has shown to be the opposite. The gut microbiome has emerged as a factor shaping metabolic responses after sweetener consumption. We examined the short-term effect of sucralose consumption on glucose homeostasis and gut microbiome of healthy male volunteers. We performed a randomised, double-blind study in thirty-four subjects divided into two groups, one that was administered sucralose capsules $(780 \mathrm{mg} / \mathrm{d}$ for $7 \mathrm{~d} ; n$ 17) and a control group receiving placebo ( $n$ 17). Before and after the intervention, glycaemic and insulinaemic responses were assessed with a standard oral glucose load ( $75 \mathrm{~g})$. Insulin resistance was determined using homeostasis model assessment of insulin resistance and Matsuda indexes. The gut microbiome was evaluated before and after the intervention by $16 \mathrm{~S}$ rRNA sequencing. During the study, body weight remained constant in both groups. Glycaemic control and insulin resistance were not affected during the 7-d period. At the phylum level, gut microbiome was not modified in any group. We classified subjects according to their change in insulinaemia after the intervention, to compare the microbiome of responders and non-responders. Independent of consuming sucralose or placebo, individuals with a higher insulinaemic response after the intervention had lower Bacteroidetes and higher Firmicutes abundances. In conclusion, consumption of high doses of sucralose for $7 \mathrm{~d}$ does not alter glycaemic control, insulin resistance, or gut microbiome in healthy individuals. However, it highlights the need to address individual responses to sucralose.

Key words: Sucralose: Non-energy artificial sweeteners: Glucose control: Insulin: Gut microbiome

Sucralose (1,6-dichloro-1,6-dideoxy- $\beta$-D-fructofuranosyl-4-chloro4-deoxy- $\alpha$-D-galactopyranoside) is a non-energy artificial sweetener (NAS) synthesised by the selective halogenation of sucrose ${ }^{(1)}$. Approved by the Food and Drug Administration for use in humans, it is 600 times sweeter than sucrose. Due to its low production cost, high thermostability and solubility, sucralose has emerged as an important sugar substitute in foods and drinks. The acceptable daily intake (ADI) of sucralose has been established at $15 \mathrm{mg} / \mathrm{kg}$ body weight $^{(2)}$.

The concept that replacing sucrose with NAS in foods and drinks improves metabolic control has been challenged ${ }^{(3)}$. In mice, sucralose added to drinking water for 11 weeks impaired oral glucose tolerance when compared with water alone or water with sucrose or glucose ${ }^{(4)}$. Such deleterious effect was prevented when mice were treated with broad-spectrum antibiotics against Gram-negative or Gram-positive bacteria. The fact that sucralose displays bacteriostatic action on several gut microbes ${ }^{(5,6)}$, and that most of the sucralose is not absorbed in the intestine ${ }^{(1,7,8)}$, gives support to observations showing that sucralose can alter gut microbiome composition ${ }^{(6,9)}$. Taken together, the notion that sucralose influences glucose control through alterations in intestinal microbiota has emerged.

In humans, consumption of high doses of sucralose for 3 months has been assessed in non-diabetic ${ }^{(10)}$ and type 2 diabetic $^{(11)}$

Abbreviation: NAS, non-energy artificial sweetener.

* Corresponding author: D. Garrido, email dgarridoc@ing.puc.cl

$\uparrow$ These authors contributed equally to this work. 
individuals. Those studies showed no influence of sucralose on glycaemic control when compared with placebo. However, betweensubject variability in given markers of glycaemic control appears higher after sucralose $v$. placebo, particularly in non-diabetic individuals. Eventually, the glycaemic response to sucralose in humans, as in mice, is also mediated through changes in gut microbiota ${ }^{(12)}$.

Considering the relevance of chronic diseases and the wide availability of sucralose in foods and drinks, it is critical to determine the effect of sucralose on metabolic responses and the gut microbiome. The goal of the present study was to evaluate the short-term effect of sucralose on glycaemic control and its interaction with the microbiota in healthy subjects.

\section{Methods \\ Subjects}

The present study was conducted in accordance with the Declaration of Helsinki and approved by the Ethics Committee of the Faculty of Medicine, Pontificia Universidad Católica de Chile. All participants provided a written informed consent. Thirty-four healthy men between 18 and 50 years with stable weight (variation $<2 \mathrm{~kg}$ in the last 3 months) and BMI between 20 and $30 \mathrm{~kg} / \mathrm{m}^{2}$ were recruited. None of them carried out intense physical activity regularly or received any drug treatment during the last 3 months. The fulfilment of all the inclusion criteria was evaluated in an initial screening, and individuals not meeting the requirements were excluded. Only males were included in order to avoid potential menstrual cycle-related changes in insulin sensitivity.

\section{Study design}

A parallel, double-blind, placebo-controlled study was performed. Selected participants were requested to fast overnight and instructed to avoid intense physical activity the day before the evaluation, in addition to smoking, alcohol and energy drinks consumption $12 \mathrm{~h}$ previous to the evaluation. On the evaluation day, subjects were requested to bring or collect in the clinical facility a faecal sample in a $15-\mathrm{ml}$ anaerobic container. The faecal sample was immediately stored at $-80^{\circ} \mathrm{C}$ for gut microbiome analysis. Body weight was measured followed by the insertion of an intravascular cannula in a peripheral vein of the arm. After resting for $30 \mathrm{~min}$, two 10-min apart blood samples were taken followed by the administration of an oral glucose load (75 g in $290 \mathrm{ml}$ solution). Blood samples were obtained after 30, 60, 90 and $120 \mathrm{~min}$ of glucose ingestion. Once finished this procedure, volunteers were randomly assigned to an intervention group with sucralose ( $n$ 17) or placebo $(n$ 17). For this we used an online resource (https://www.randomizer.org/). Randomisation was conducted by one of the members of our group. This person was not involved in recruiting, selecting or performing any of the measurements of the present study, and broke the labels after data analysis. Four subjects did not finish the study, resulting in sixteen subjects in the sucralose group and fourteen in the placebo group. Individuals were instructed to ingest one capsule containing sucralose or placebo, three times a day for 7 d. Sucralose was purchased from VitaSweet. Each sucralose capsule contained $260 \mathrm{mg}$ sucralose and $70 \mathrm{mg}$ calcium carbonate; thus the three capsules consumed daily were equivalent to the $75 \%$ of the acceptable daily intake ${ }^{(2)}$ $(15 \mathrm{mg} / \mathrm{kg}$ per d) of a subject weighing $70 \mathrm{~kg}$. Each placebo capsule contained $250 \mathrm{mg}$ of calcium carbonate. Each subject was instructed to register and report any adverse events. After $7 \mathrm{~d}$, subjects were requested to attend the clinical facility to repeat the same procedures. Volunteers were instructed to record any adverse events or change in their customary diet.

\section{Glucose and insulin tests}

Plasma concentrations of glucose were determined by the glucose oxidase method, in a dry chemistry equipment (Vitros 4600, Ortho Clinical Diagnostics). Serum concentrations of insulin were quantified by a capture chemiluminescent immunoassay (Centaur XPT, Siemens). Insulin resistance was calculated as the homeostasis model assessment of insulin resistance index, using the formula by Matthews et al. ${ }^{(13)}$ : (fasting insulin $(\mu \mathrm{U} / \mathrm{ml}) \times$ fasting glucose $(\mathrm{mmol} / \mathrm{l})) / 22.5$. Alternatively, the insulin sensitivity index (ISI)-composite was also determined, using the formula proposed by Matsuda \& DeFronzo $^{(14)}: 10$ 000/(fasting glycaemia $(\mathrm{mg} / \mathrm{dl}) \times$ fasting insulin $(\mu \mathrm{U} / \mathrm{ml}) \times$ average glycaemia in the oral test $(30-120 \mathrm{~min})(\mathrm{mg} /$ $\mathrm{dl}) \times$ average insulin in the oral test $(30-120 \mathrm{~min})(\mu \mathrm{U} / \mathrm{ml}))^{0.5}$. Total AUC for insulinaemic and glycaemic responses were also calculated, using the trapezoidal rule $\mathrm{e}^{(15)}$.

\section{Statistical analysis}

Clinical data were expressed as averages and standard deviations. Statistical analyses were run on SASv9.2 (SAS Institute). Subjects' characteristics at screening were compared between groups using Student's $t$ test. Differences in the AUC for the glycaemic and insulinaemic responses were assessed through the Wilcoxon's rank sums test and the Mann-Whitney $U$ test. Differences in clinical variables before and after the intervention were determined using a repeated-measures ANOVA including group, time (repeated) and the group $\times$ time interaction. The significance level for all analyses was set at $P<0 \cdot 05$.

\section{Sample size}

The sample size needed to detect a difference in glycaemia (i.e. mean of glycaemia between 30 and 120 min after oral glucose tolerance test) after 1-week sucralose ingestion was estimated from two 4-week apart oral glucose tolerance tests performed in healthy, young, non-obese individuals ${ }^{(16)}$. Considering a withinsubject standard deviation of $\pm 11 \mathrm{mg} / \mathrm{dl}(0.61 \mathrm{mmol} / \mathrm{l})$ as the expected variability without intervention, and a between-subject standard deviation of $21 \mathrm{mg} / \mathrm{dl}(1.17 \mathrm{mmol} / \mathrm{l})$, fifteen subjects will allow detecting a difference of $13 \mathrm{mg} / \mathrm{dl}(0.72 \mathrm{mmol} / \mathrm{l})$ in glycaemia after 1 -week sucralose ingestion, with a power of $82 \%$ for paired samples two-sided and significance level of $5 \%$.

\section{Gut microbiome analysis}

Faecal samples stored at $-80^{\circ} \mathrm{C}$ were first thawed and $150 \mathrm{mg}$ were used for total DNA extraction (Quick-DNA Fecal/Soil Microbe Miniprep Kit; Zymo Research) using a Disruptor Genie device (Scientific Industries) $^{(17)}$. DNA samples were quantified 
Table 1. Clinical parameters at screening (Mean values, standard deviations and ranges)

\begin{tabular}{|c|c|c|c|c|c|c|c|}
\hline & \multicolumn{3}{|c|}{ Placebo ( $n 14)$} & \multicolumn{3}{|c|}{ Sucralose ( $n$ 16) } & \multirow[b]{2}{*}{$P(t$ test $)$} \\
\hline & Mean & SD & Range & Mean & SD & Range & \\
\hline Age (years) & 23.5 & $2 \cdot 9$ & $18 \cdot 2,29 \cdot 3$ & $22 \cdot 8$ & 3.0 & $18 \cdot 7,30 \cdot 2$ & 0.51 \\
\hline Weight (kg) & $77 \cdot 0$ & $8 \cdot 3$ & $57 \cdot 9,88 \cdot 0$ & 73.2 & 6.9 & $60 \cdot 9,83 \cdot 7$ & 0.19 \\
\hline Height $(m)$ & 1.73 & 0.04 & $1.67,1.80$ & 1.75 & 0.07 & $1.63,1.90$ & 0.31 \\
\hline BMI $\left(\mathrm{kg} / \mathrm{m}^{2}\right)$ & $25 \cdot 7$ & 2.9 & $20 \cdot 8,28.9$ & $23 \cdot 8$ & 1.7 & $21 \cdot 1,26 \cdot 6$ & 0.04 \\
\hline Glycaemia $(\mathrm{mg} / \mathrm{dl})^{\star}$ & 84 & 8 & 67,95 & 85 & 6 & 72,94 & 0.50 \\
\hline Cholesterol $(\mathrm{mg} / \mathrm{dl})^{*}$ & 173 & 23 & 134,219 & 147 & 22 & 106,184 & $<0.01$ \\
\hline
\end{tabular}

* To convert glycaemia in $\mathrm{mg} / \mathrm{dl}$ to $\mathrm{mmol} / \mathrm{l}$, multiply by 0.0555 . To convert cholesterol in $\mathrm{mg} / \mathrm{dl}$ to $\mathrm{mmol} / \mathrm{l}$, multiply by 0.0259 .

Table 2. Metabolic response to intervention

(Mean values and standard deviations)

\begin{tabular}{|c|c|c|c|c|c|c|c|c|c|c|c|c|c|c|c|}
\hline & \multicolumn{6}{|c|}{ Placebo $(n 14)$} & \multicolumn{6}{|c|}{ Sucralose $(n 16)$} & \multirow{2}{*}{\multicolumn{3}{|c|}{$P$}} \\
\hline & \multicolumn{2}{|c|}{ Before } & \multicolumn{2}{|c|}{ After } & \multicolumn{2}{|c|}{ Change* $^{\star}$} & \multicolumn{2}{|c|}{ Before } & \multicolumn{2}{|c|}{ After } & \multicolumn{2}{|c|}{ Change } & & & \\
\hline & Mean & SD & Mean & SD & Mean & SD & Mean & SD & Mean & SD & Mean & SD & Group & Time & Group xtime \\
\hline \multicolumn{16}{|l|}{ Fasting } \\
\hline Glycaemia (mg/dl)† & 82 & 5 & 79 & 4 & $-2 \cdot 2$ & $5 \cdot 0$ & 82 & 5 & 82 & 5 & 0.0 & $6 \cdot 1$ & 0.22 & 0.31 & 0.29 \\
\hline Insulinaemia $(\mu \mathrm{U} / \mathrm{ml})$ & 12 & 5 & 11 & 4 & $-1 \cdot 0$ & 3.3 & 9 & 4 & 8 & 4 & -0.9 & 4.6 & 0.07 & 0.22 & 0.93 \\
\hline HOMA-IR & $2 \cdot 4$ & $1 \cdot 1$ & $2 \cdot 1$ & 0.8 & -0.3 & 0.7 & 1.9 & 0.9 & $1 \cdot 7$ & 0.9 & -0.2 & $1 \cdot 1$ & $0 \cdot 13$ & $0 \cdot 21$ & 0.77 \\
\hline \multicolumn{16}{|l|}{ After oral glucose } \\
\hline Glycaemia (mg/dl)†‡ & 115 & 17 & 112 & 21 & $-3 \cdot 0$ & $17 \cdot 4$ & 107 & 21 & 113 & 21 & $6 \cdot 2$ & $18 \cdot 6$ & 0.65 & 0.63 & 0.17 \\
\hline Insulinaemia $(\mu \mathrm{U} / \mathrm{ml}) \ddagger$ & 81 & 38 & 87 & 51 & $5 \cdot 6$ & $38 \cdot 1$ & 63 & 40 & 78 & 41 & $15 \cdot 4$ & 30.5 & 0.35 & $0 \cdot 11$ & 0.44 \\
\hline ISI-composite & $4 \cdot 1$ & $2 \cdot 1$ & 4.7 & 3.0 & 0.5 & $2 \cdot 4$ & $7 \cdot 8$ & $10 \cdot 0$ & 5.9 & 4.2 & -1.9 & $7 \cdot 7$ & 0.21 & 0.52 & 0.29 \\
\hline
\end{tabular}

HOMA-IR, homeostasis model assessment of insulin resistance; ISI, insulin sensitivity index.

* Calculated as values after minus before intervention.

† To convert glycaemia in $\mathrm{mg} / \mathrm{dl}$ to $\mathrm{mmol} / \mathrm{l}$, multiply by 0.0555 .

$\ddagger$ Mean of the respective response over the $30-120$ min period after glucose ingestion.

(NanoDrop 2000c; Thermo Fisher Scientific USA) and diluted to $20 \mathrm{ng} / \mu \mathrm{l}$ in nuclease-free water (IDT). DNA samples were submitted for Illumina MiSeq sequencing at Integrated Microbiome Resource. The 16S rRNA gene V3-V4 variable region was amplified using V3-V4 primers $^{(18)}$, which also included a barcode in the forward primer. The DADA2 v1.10 R package was used to analyse the $16 \mathrm{~S}$ rRNA gene sequences ${ }^{(19)}$, following a modified procedure $^{(19)}$. Briefly, the sequences were filtered by quality and trimmed to remove the barcode and low-quality nucleotides prior to estimating the sequencing error. Then, sequences were denoised to identify Amplicon Sequence Variants ${ }^{(20)}$, then merged and used to assign microbial taxonomy following a naïve Bayesian classifier $^{(21)}$, employing the SILVA database version $132^{(22,23)}$. Raw data paired-end reads obtained from the MiSeq platform were stored in the European Nucleotide Archive database, under study accession number PRJEB27704.

\section{Bioinformatic analysis}

Microbiome composition similarity at phylum level was determined employing principal coordinates analysis with distances calculated with the weighted UniFrac method ${ }^{(24,25)}$. The weighted UniFrac quantitatively estimates the $\beta$-diversity taking into account phylogenetic distances between microbial taxa and their relative abundance. The similarity between microbiome compositions was calculated with the unweighted pair group method with arithmetic mean algorithm. Fold changes in the relative microbiome composition at the phylum level (values after/before intervention), for each group of subjects and subgroups, were determined using the Mann-Whitney $U$ test. The significance level for all analyses was set at $P<0 \cdot 05$.

\section{Results}

\section{Subjects characteristics at the screening}

Age, weight and height were similar between groups (Table 1), while BMI was higher in the placebo compared with the sucralose group $(P=0 \cdot 04)$. Such difference in BMI was accompanied by higher blood cholesterol concentration $(P<0 \cdot 01)$, but similar blood glucose concentration (Table 1).

\section{Metabolic responses to sucralose}

Body weight remained stable throughout the study, with an average change of $0.16(\mathrm{SD} 0.74) \mathrm{kg}(P=0.44)$ and $0.21(\mathrm{SD} 1.17) \mathrm{kg}$ $(P=0.49)$ in the placebo and sucralose groups, respectively. Volunteers in the placebo and sucralose groups did not report adverse events or changes in their usual dietary pattern. As observed at screening, fasting plasma glucose concentration was similar between groups and not affected by the consumption of placebo or sucralose (Table 2). In turn, a borderline higher fasting serum insulin concentration was observed in the placebo $v$. sucralose group $(P=0 \cdot 07$; Table 2$)$. Similar to 
(a)
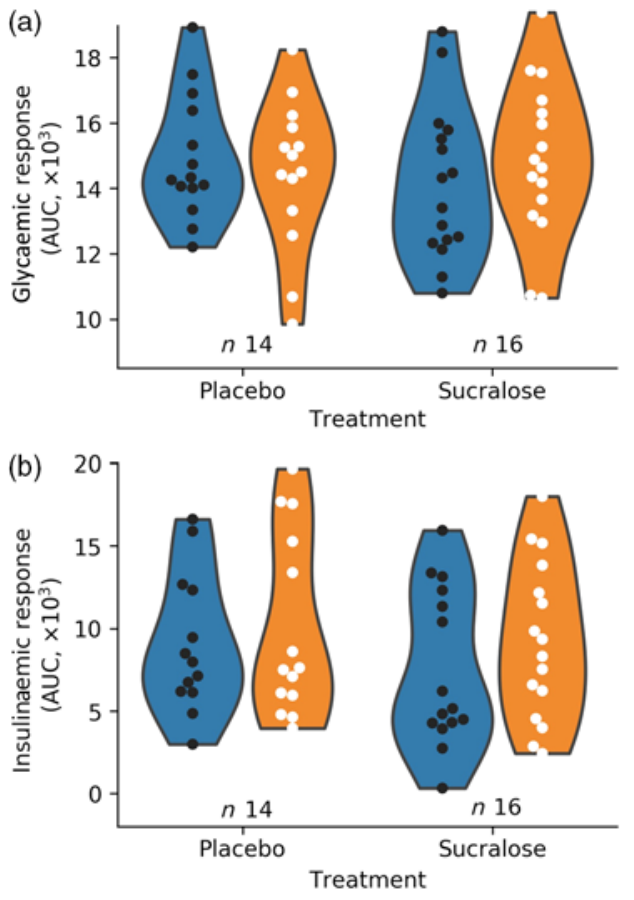

Fig. 1. Changes in metabolic responses upon oral glucose consumption before and after the intervention. (a) Total glycaemic AUC for each group $(P=0.57)$; (b) insulinaemic total AUC $(P=0.73)$. Subjects with insufficient data to calculate the AUC were not included. The kernel density estimation shows the probability of the values.

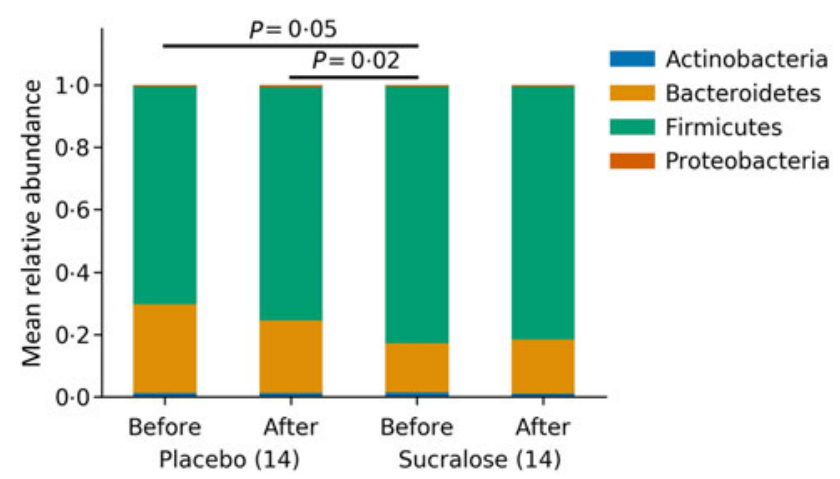

Fig. 2. Gut microbiome compositions for each group before and after each treatment. Bars show the average relative abundance of the four dominant phyla of the human gut microbiome.

fasting plasma glucose, consumption of sucralose or placebo did not affect fasting serum insulin concentration (Table 2).

Upon glucose ingestion, the average glycaemic and insulinaemic responses were similar between groups and not affected by the consumption of sucralose or placebo (Table 2). Regarding insulin resistance (sensitivity) markers, no differences by group and intervention were detected (Table 2).

One further assessment included the analysis of the changes in the aforementioned variables after both interventions. Consistent with ANOVA, none of the changes was different from zero (Table 2). We calculated the AUC for glycaemic and insulinaemic responses for each subject (Fig. 1). This analysis did not show alterations in glycaemia or insulinaemia in any group.
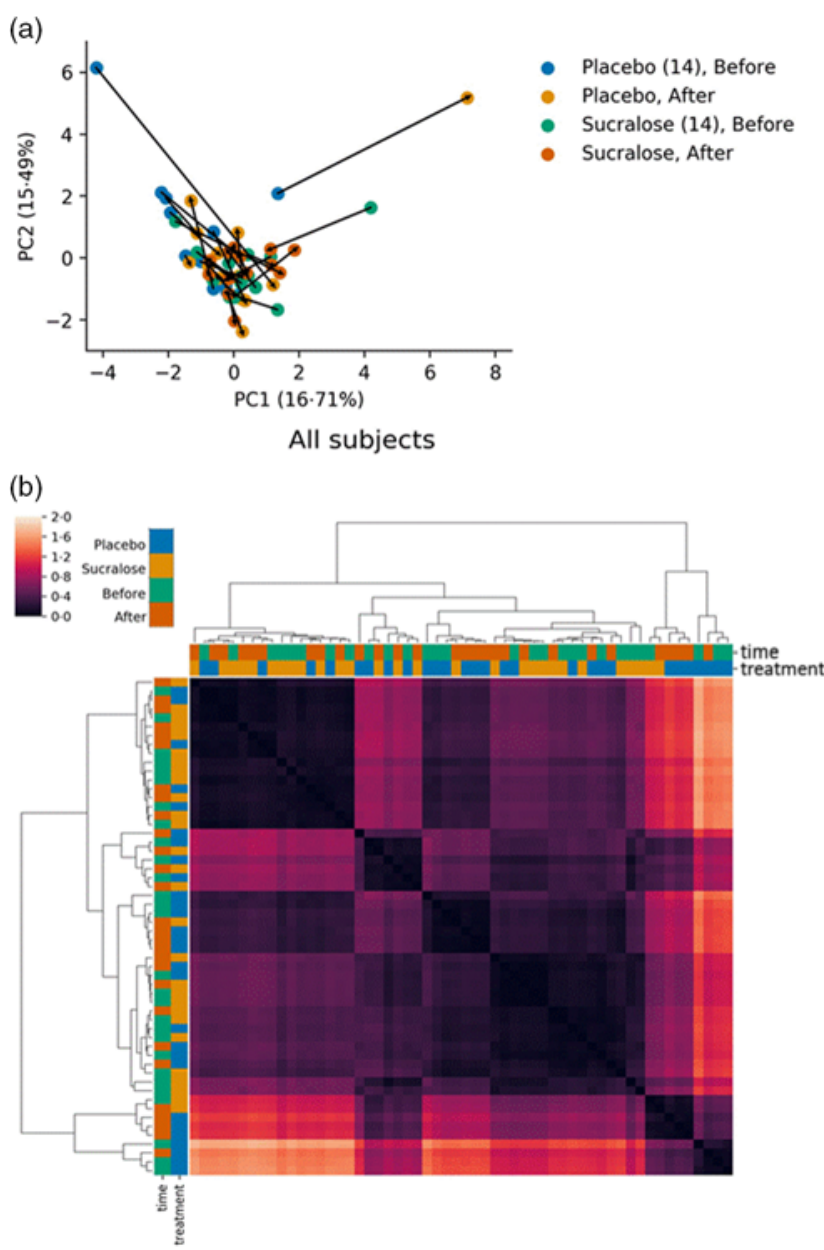

Fig. 3. Comparisons of gut microbiome composition between subjects. (a) Principal coordinates analysis of all phyla identified showing close similarity for groups including both placebo and sucralose at both intervention times. Arrows show the trajectories of changes in microbiome composition for each individual in the study. (b) Heatmap of distances calculated as the weighted UniFrac and clustering of closest distances using the UPMGA algorithm.

\section{Changes in gut microbiome composition}

The gut microbiome of all subjects in each group, both before and after the 7-d intervention period, was analysed. As expected, the Firmicutes and Bacteroidetes phyla were dominant in the microbiome of these subjects (online Supplementary Fig. S1). Smaller representations of Actinobacteria, Verrucomicrobia and Proteobacteria were also observed. On average, a higher relative abundance of the Firmicutes phylum in the placebo $v$. sucralose group was observed before initiating the treatments (Fig. 2). However, microbiome composition remained stable throughout in both interventions (Fig. 2).

We performed a principal component analysis to identify variations in the gut microbiome composition for each individual before and after interventions (Fig. 3(a)). This principal component analysis represents the normalised absolute abundance for all fourteen phyla identified in every microbiome sample evaluated. Consistent with the aforementioned analysis, most subjects displayed modest variations in their microbiome composition (Fig. 3(a)). 
(a)

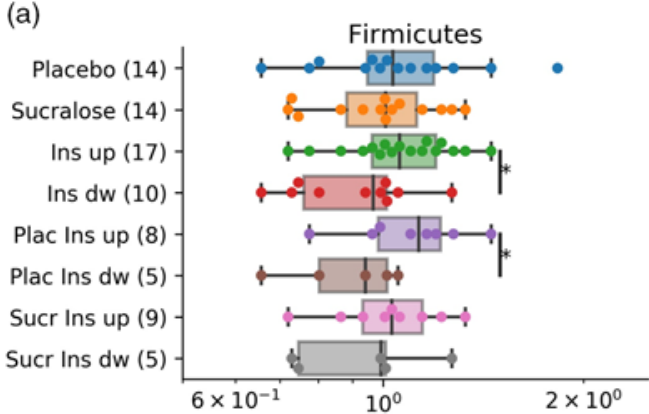

(c)

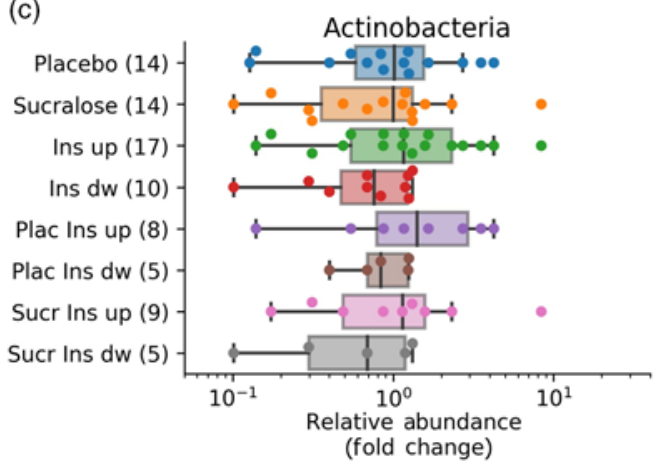

(b)

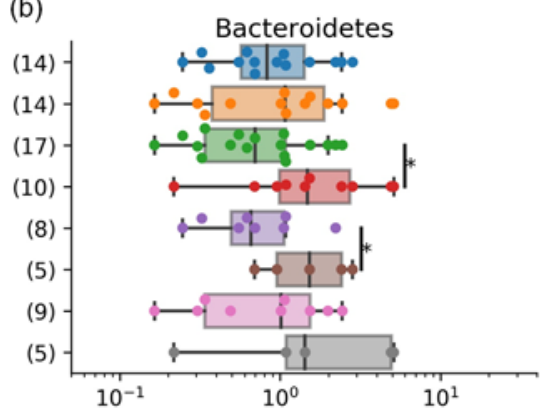

(d)

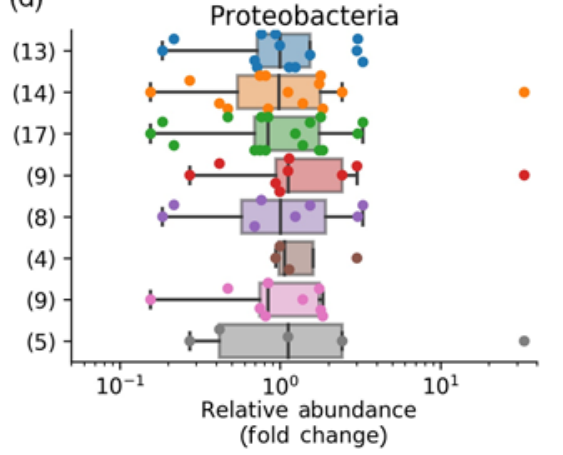

Fig. 4. Pairwise correlations between fold changes in microbiome phyla and insulinaemia responder status. The figure shows the fold change in all major four phyla before and after the intervention, correlated with subgroups on the x-axis. Insulinaemia AUC ratios were calculated with AUC values after/before the intervention. (a) Fold changes in Firmicutes; (b) Bacteroidetes; (c) Actinobacteria; (d) Proteobacteria. Boxplots indicate the median and the interquartile range, with whiskers determined as the 1.5 range of the box. * Significant differences $(P<0.05)$ determined with the Mann-Whitney $U$ test. Ins, insulinaemia; dw, down; Plac, placebo; Sucr, sucralose.

We also calculated the weighted UniFrac distances for each gut microbiome (Fig. 3(b)), in order to determine biological diversity among the microbiomes. This analysis showed that microbiomes in the sucralose group before and after the intervention (S1/S2) tend to cluster together, as well as microbiomes from subjects in the placebo group before and after the intervention $(\mathrm{P} 1 / \mathrm{P} 2)$. Again, this analysis indicates that both treatments did not substantially modify the microbiome of these subjects, while differences detected before interventions remained.

\section{Correlations between gut microbiome and metabolic markers}

Next, we classified individuals according to their metabolic responses, by calculating the after-to-before ratio for serum insulin and glucose AUC, BMI and insulin resistance (sensitivity) markers. This was aimed to determine if within the same treatment certain subjects responded differently, identifying responders (subjects with a ratio higher than 1) and nonresponders (a ratio smaller or equal to 1$)^{(4)}$.

Independent of the treatment received, responders $v$. nonresponders according to insulin AUC had higher Firmicutes and lower Bacteroidetes abundances in their microbiomes (Fig. 4(a) and (b)). Such differences were also noted when classifying subjects according to their change in glucose AUC, albeit with borderline significance (online Supplementary Fig. S2). Bacteroidetes relative abundance was also lower in responders according to changes in homeostasis model assessment (online Supplementary Fig. S3). Finally, classifying individuals by their
BMI (overweight or normal weight) did not show significant changes in their gut microbiomes regardless of the treatment received. Only for individuals in the placebo group, we observed that overweight individuals had higher Firmicutes and Actinobacteria compared with those with normal weight (online Supplementary Fig. S4).

\section{Discussion}

\section{Evidence of metabolic impairments for non-energy artificial sweetener}

Sucralose is one of the most consumed NAS in the world ${ }^{(26)}$, with several foods and beverages being supplemented with this sweetener. While recognised as safe by several studies, recent evidence has shown that sucralose among other NAS may promote weight gain and metabolic disturbances such as glucose intolerance ${ }^{(27)}$. Therefore, it is critical to understand the actual impact of NAS in our metabolism.

Previous findings in mice showed no adverse effects of sucralose on inflammatory markers or fasting glucose levels, even at large doses for up to 2 years ${ }^{(28,29)}$. A study in diabetic subjects administrating sucralose at a dose of $667 \mathrm{mg}$ (about $7.5 \mathrm{mg} / \mathrm{kg}$ per d) for 13 weeks did not observe changes in glycated $\mathrm{Hb}$, fasting glycaemia or fasting C-peptide against placebo $^{(11)}$, which are similar observations compared with this work. Another study conducted in non-diabetic individuals who consumed $1000 \mathrm{mg} / \mathrm{d}$ of sucralose (about $13.2 \mathrm{mg} / \mathrm{kg}$ per d) for 12 weeks did not detect differences relative to placebo in fasting glycaemia, insulinaemia or glycated $\mathrm{Hb}^{(10)}$. Sucralose consumption at the population level 
may reach up to $15 \%$ acceptable daily intake, which is evidently lower compared with the amount provided in these previous reports ${ }^{(30)}$. None of these studies evaluated the gut microbiome composition of these subjects. Here we performed a short-term study with small sample size, finding that sucralose consumption at high doses does not alter the glycaemic response of healthy individuals.

Recently the gut microbiome has emerged as a factor that could contribute to the biological effects of NAS, in particular sucralose ${ }^{(31)}$. Early environmental studies revealed a strong bacteriostatic effect of several NAS, including sucralose and saccharine $^{(5,6,31,32)}$. Wang et al. ${ }^{(6)}$ also showed increases in Firmicutes in mice exposed to sucralose. Thus, alterations in gut microbiome have been shown to be associated with NAS exposure, and these alterations could be causative of metabolic impairments such as glucose intolerance and insulin resistance ${ }^{(4)}$. In C57/BL6 mice sucralose altered microbiome composition, increased proinflammatory faecal metabolites and induced hepatic proinflammatory markers ${ }^{(33)}$. Suez et al. ${ }^{(4)}$ reported that in addition to saccharin, sucralose induced glucose intolerance in mice. For saccharin, this metabolic effect is transmittable to germ-free mice through their gut microbiome, indicating causality ${ }^{(4)}$.

The Suez et al. ${ }^{(4)}$ study was pioneer in showing that after short-term saccharin exposure, individuals display contrasting responses in terms of glucose tolerance and microbiome composition. Some of the observations raised have, however, been questioned by other studies ${ }^{(12)}$. Interestingly, that contrasting glucose tolerance was emulated in germ-free mice transplanted with the microbiome of the human donors. That study also provided insights into what micro-organisms are being altered in the complex gut microbiome. Saccharin consumption in the short term resulted in enrichment in the Bacteroides phylum, especially Bacteroides vulgatus and Bacteroides fragilis $^{(4)}$. Interestingly this exposure in mice also resulted in decreased abundance of Akkermansia muciniphila, a key gut microbe that has been associated with anti-inflammatory properties ${ }^{(34,35)}$. While short-term saccharin consumption resulted in an increase in Bacteroidales and decrease in Clostridiales in responders ${ }^{(4)}$, here we observed that sucralose administration at $75 \%$ of the maximum acceptable daily intake for a similar period of time resulted in no major changes in the gut microbiome composition in healthy subjects. Evidently, metabolic and microbiome responses to saccharin could be very different compared with those of sucralose, considering their chemical properties and their differences in absorption and metabolism. The absence of the effect of sucralose consumption on gut microbiome composition could be explained for powering the study on glycaemia and not on gut microbiome data.

We observed that at the beginning of the study, subjects in the placebo group had a different gut microbiome (higher Firmicutes and lower Bacteroidetes) compared with the sucralose group. Interestingly this correlated with these subjects having higher initial BMI and cholesterol compared with the sucralose group. These differences were not intentional and could be expected from the randomisation of a population of healthy subjects. Moreover, subjects in the placebo group with higher insulinaemia, homeostasis model assessment or BMI presented significant changes in their gut microbiomes (Fig. 4, online Supplementary Figs. S3 and S4). These changes were a higher abundance of the Firmicutes phylum and lower Bacteroidetes. These observations indicate that the placebo group had substantial differences regarding glycaemic response and gut microbiome compared with the sucralose group, and even inside the placebo group there were interesting differences between these individuals. In spite of these baseline differences, neither group presented changes in glycaemic response or gut microbiome associated with the treatment received.

In addition, we did not observe differences in the gut microbiome between responders and non-responders associated with sucralose or placebo consumption, using criteria changes in serum insulin AUC. However, subjects who had a higher insulin AUC after the intervention, and regardless of the treatment received, had a higher Firmicutes:Bacteroidetes ratio (Fig. 4). This indicates that this metabolic difference could be more relevant than the intervention itself. This also correlates with the idea of this cohort being so healthy that only minimal or very sensitive changes in the gut microbiome were obtained. Changes in these phyla are probably relevant and implicate a considerable rearrangement of the community, considering they represent more than $90 \%$ of the total gut microbiome. This same phenotype (higher Firmicutes and lower Bacteroidetes, or a higher Firmicutes:Bacteroidetes ratio) has been observed in several studies reporting alterations in the gut microbiome in obese subjects and especially in type 2 diabetes ${ }^{36-39)}$. Mechanistically, these microbiomes have been associated with low-grade inflammation, higher gut permeability and higher circulating lipopolysaccharide ${ }^{(40)}$.

\section{Conclusion}

The present study shows that the consumption of high doses of sucralose for $7 \mathrm{~d}$ in healthy subjects does not alter glycaemic control. There were no changes in the gut microbiomes of these subjects with respect to the consumption of sucralose or placebo. Independent of the intervention, subjects displaying an increase $v$. decrease in insulinaemia after either intervention had different gut microbiome compositions. Thus, initial metabolic differences could have been more important than the intervention itself in terms of altering the gut microbiome. Further studies should study the impact of other important non-energy sweeteners, but including potential responder-non-responder differences among subjects.

\section{Acknowledgements}

We appreciate the contribution to this work of Angélica Ubilla, Daniel Medina, Verónica Ortúzar, Camila Solar and Carolina Ulloa.

This project was funded by VRI-UC, Concurso de Investigación Interdisciplinaria 2015, VRI 13/2015 (C. A., J. E. G., D. G.) and Fondecyt Postdoctorado 3170609 (P. T.).

The authors declare no conflict of interest.

\section{Supplementary material}

For supplementary material/s referred to in this article, please visit https://doi.org/10.1017/S0007114519001570 


\section{References}

1. Magnuson BA, Carakostas MC, Moore NH, et al. (2016) Biological fate of low-calorie sweeteners. Nutr Rev 74, 670-689.

2. Martyn D, Darch M, Roberts A, et al. (2018) Low-/no-calorie sweeteners: a review of global intakes. Nutrients 10, 357.

3. Shearer J \& Swithers SE (2016) Artificial sweeteners and metabolic dysregulation: lessons learned from agriculture and the laboratory. Rev Endocr Metab Disord 17, 179-186.

4. Suez J, Korem T, Zeevi D, et al. (2014) Artificial sweeteners induce glucose intolerance by altering the gut microbiota. Nature 514, 181-186.

5. Omran A, Ahearn G, Bowers D, et al. (2013) Metabolic effects of sucralose on environmental bacteria.J Toxicol 2013, 372986.

6. Wang Q-P, Browman D, Herzog H, et al. (2018) Non-nutritive sweeteners possess a bacteriostatic effect and alter gut microbiota in mice. PLOS ONE 13, e0199080.

7. Sylvetsky AC, Bauman V, Blau JE, et al. (2017) Plasma concentrations of sucralose in children and adults. Toxicol Environ Chem 99, 535-542.

8. Roberts A, Renwick AG, Sims J, et al. (2000) Sucralose metabolism and pharmacokinetics in man. Food Chem Toxicol 38, 31-41.

9. Rodriguez-Palacios A, Harding A, Menghini P, et al. (2018) The artificial sweetener Splenda promotes gut proteobacteria, dysbiosis, and myeloperoxidase reactivity in Crohn's disease-like ileitis. Inflamm Bowel Dis 24, 1005-1020.

10. Grotz VL, Pi-Sunyer X, Porte D, et al. (2017) A 12-week randomized clinical trial investigating the potential for sucralose to affect glucose homeostasis. Regul Toxicol Pharmacol 88, 22-33.

11. Grotz VL, Henry RR, McGill JB, et al. (2003) Lack of effect of sucralose on glucose homeostasis in subjects with type 2 diabetes. J Am Diet Assoc 103, 1607-1612+1711.

12. Lobach AR, Roberts A \& Rowland IR (2019 Assessing the in vivo data on low/no-calorie sweeteners and the gut microbiota. Food Chem Toxicol 124, 385-399.

13. Matthews DR, Hosker JP, Rudenski AS, et al. (1985) Homeostasis model assessment: insulin resistance and $\beta$-cell function from fasting plasma glucose and insulin concentrations in man. Diabetologia 28, 412-419.

14. Matsuda M \& DeFronzo RA (1999) Insulin sensitivity indices obtained from oral glucose tolerance testing: comparison with the euglycemic insulin clamp. Diabetes Care 22, 1462-1470.

15. Krzyzanski W \& Jusko WJ (1998) Integrated functions for four basic models of indirect pharmacodynamic response. J Pharm Sci 87, 67-72.

16. Galgani JE, Vasquez K, Watkins G, et al. (2013) Enhanced skeletal muscle lipid oxidative efficiency in insulin-resistant vs insulin-sensitive nondiabetic, nonobese humans. J Clin Endocrinol Metab 98, E646-E653.

17. Medina DA, Pedreros JP, Turiel D, et al. (2017) Distinct patterns in the gut microbiota after surgical or medical therapy in obese patients. PeerJ 2017, e3443.

18. Comeau AM, Douglas GM \& Langille MGI (2017) Microbiome helper: a custom and streamlined workflow for microbiome research. mSystems 2, e00127-16.

19. Callahan BJ, McMurdie PJ, Rosen MJ, et al. (2016) DADA2: high-resolution sample inference from Illumina amplicon data. Nat Methods 13, 581-583.

20. Callahan BJ, McMurdie PJ \& Holmes SP (2017) Exact sequence variants should replace operational taxonomic units in markergene data analysis. ISME J 11, 2639-2643.
21. Wang Q, Garrity GM, Tiedje JM, et al. (2007) Nave Bayesian classifier for rapid assignment of rRNA sequences. Appl Environ Microbiol 73, 5261-5267.

22. Quast C, Pruesse E, Yilmaz P, et al. (2013) The SILVA ribosomal RNA gene database project: improved data processing and web-based tools. Nucleic Acids Res 41, D590-D596.

23. Schweer T, Parfrey LW, Quast C, et al. (2013) The SILVA and 'All-species Living Tree Project (LTP)' taxonomic frameworks. Nucleic Acids Res 42, D643-D648.

24. Lozupone CA, Hamady M, Kelley ST, et al. (2007) Quantitative and qualitative $\beta$ diversity measures lead to different insights into factors that structure microbial communities. Appl Environ Microbiol 73, 1576-1585.

25. Lozupone C, Lladser ME, Knights D, et al. (2011) UniFrac: an effective distance metric for microbial community comparison. ISME J. 5, 169-172.

26. Schiffman SS \& Rother KI (2013) Sucralose, a synthetic organochlorine sweetener: overview of biological issues. $J$ Toxicol Environ Heal B Crit Rev 16, 399-451.

27. Swithers SE (2013) Artificial sweeteners produce the counterintuitive effect of inducing metabolic derangements. Trends Endocrinol Metab 24, 431-441.

28. Johannsen FR \& Levinskas GJ (1987) Acute and subchronic toxicity of tetramethylcyclohexanes. J Appl Toxicol 7, 245-248.

29. Grice HC \& Goldsmith LA (2000) Sucralose - an overview of the toxicity data. Food Chem Toxicol 38, 1-6.

30. Magnuson BA, Roberts A \& Nestmann ER (2017) Critical review of the current literature on the safety of sucralose. Food Chem. Toxicol 106, 324-355.

31. Suez J, Korem T, Zilberman-Schapira G, et al. (2015) Noncaloric artificial sweeteners and the microbiome: findings and challenges. Gut Microbes 6, 149-155.

32. Omran A, Baker R \& Coughlin C (2013) Differential bacteriostatic effects of sucralose on various species of environmental bacteria. ISRN Toxicol 2013, 415070

33. Bian X, Chi L, Gao B, et al. (2017) Gut microbiome response to sucralose and its potential role in inducing liver inflammation in mice. Front Physiol 8, 487.

34. Zhao S, Liu W, Wang J, et al. (2017) Akkermansia muciniphila improves metabolic profiles by reducing inflammation in chow diet-fed mice. J Mol Endocrinol 58, 1-14.

35. Everard A, Belzer C, Geurts L, et al. (2013) Cross-talk between Akkermansia muciniphila and intestinal epithelium controls diet-induced obesity. Proc Natl Acad Sci U S A 110, 9066-9071.

36. Most J, Goossens GH, Reijnders D, et al. (2017) Gut microbiota composition strongly correlates to peripheral insulin sensitivity in obese men but not in women. Benef Microbes 8, 557-562.

37. Sircana A, Framarin L, Leone N, et al. (2018) Altered gut microbiota in type 2 diabetes: just a coincidence? Curr Diab Rep 18, 98.

38. Kasai C, Sugimoto K, Moritani I, et al. (2015) Comparison of the gut microbiota composition between obese and non-obese individuals in a Japanese population, as analyzed by terminal restriction fragment length polymorphism and next-generation sequencing. BMC Gastroenterol 15, 100.

39. Ley RE, Mardis ER, Magrini V, et al. (2006) An obesityassociated gut microbiome with increased capacity for energy harvest. Nature 444, 1027-1031.

40. Boulange CL, Neves AL, Chilloux J, et al. (2016) Impact of the gut microbiota on inflammation, obesity, and metabolic disease. Genome Med $\mathbf{8}, 42$. 